\title{
Primary hyperparathyroidism
}

\author{
Hafsah Al-Azem HBSc, Aliya Khan MD
}

\section{The case}

A 17-year-old man presented at the clinic with thirst, lethargy and fatigue that had been ongoing for several months. His family physician had noted hypercalcemia, and the patient had been referred for an endocrine evaluation and treatment. His appetite and weight were stable, and his only gastrointestinal complaint was constipation. He was a nonsmoker and was otherwise healthy. He had no history of fractures or kidney stone. The physical examination was unremarkable. His blood pressure was $130 / 60 \mathrm{~mm}$ $\mathrm{Hg}$ and his heart rate was 60 beats/min.

Laboratory investigations on three occasions over a one-month period showed that the patient's serum level of calcium (adjusted for albumin) was $2.67-2.88 \mathrm{mmol} / \mathrm{L}$, his level of ionized calcium was $1.45-1.50 \mathrm{mmol} / \mathrm{L}$, and his level of parathyroid hormone was 5.50$10.3 \mathrm{pmol} / \mathrm{L}$. The results of the laboratory investigations are presented in Table 1.

$\mathrm{H}$ ypercalcemia may be detected incidentally in a person who is otherwise well or during investigations in a patient with symptoms. Although many conditions can cause hypercalcemia, the most common cause for hypercalcemia in a patient with mild or no symptoms is primary hyperparathyroidism.

The first part of this primer provides a general outline for the investigation of hypercalcemia, using the case of our patient as an example. The second part focuses on the management of primary hyperparathyroidism, and is based on recommendations from the Third International Workshop on the Management of Asymptomatic Primary Hyperparathyroidism, an international collaborative effort of experts in parathyroid disease.

\section{Is it true hypercalcemia?}

Because $45 \%$ of the calcium in the serum is bound to protein (usually albumin), the concentra- tion of serum calcium is adjusted using the following calculation: corrected calcium $=$ measured calcium $+(40-$ measured albumin $\times 0.02)$, where corrected and measured calcium concentrations are presented in millimoles per litre, and measured albumin is presented in grams per litre. This correction excludes fictitious elevations in calcium due to hyperalbuminemia.

Measuring the concentration of ionized calcium in the serum allows the physician to exclude pseudohypercalcemia. Pseudohypercalcemia can occur in the presence of calciumbinding paraproteins. In vitro, it can be caused by thrombocythemia with the release of intracellular calcium. The concentration of ionized calcium in the serum should be measured without using a tourniquet after the patient has fasted, and the measurement should be repeated before hypercalcemia is confirmed.

\section{What causes hypercalcemia?}

Incidental detection of hypercalcemia in an otherwise healthy individual is most commonly due to primary hyperparathyroidism. In patients who are ill or admitted to hospital, the most common cause of hypercalcemia is a malignant growth (> 50\% of patients) $;{ }^{1}$ however, the underlying tumour is usually clinically evident before hypercalcemia is seen. A careful history and physical examination in combination with labo-

\section{KEY POINTS}

- Other conditions that mimic primary hyperparathyroidism should be excluded before the diagnosis can be confirmed.

- Imaging studies alone should not be used to confirm a diagnosis of primary hyperparathyroidism; however, they are useful guides to surgery.

- A parathyroidectomy in patients with asymptomatic disease when there are no contraindications has been associated with decreased risk of fracture and increases in bone density.

- New surgical approaches include minimally invasive parathyroidectomy, which has rates of success approaching those seen with the traditional method. 
ratory investigation ${ }^{1-3}$ will help to determine the most likely cause of hypercalcemia (Box 1). The differential diagnosis of hypercalcemia based on the level of parathyroid hormone in the serum is outlined in Box 2. 1,3,4

When a malignant growth is clinically suspected, appropriate imaging and a search for the growth should be done regardless of the serum level of parathyroid hormone. Hyperparathyroidism can occur concomitantly with an occult malignant growth, and hypercalcemia may sometimes be due to the ectopic secretion of parathyroid hormone by cancer cells. ${ }^{4}$ Initial imaging may include a radiograph of the chest, a bone scan, a mammogram, and ultrasounds of the abdomen and pelvis, as well as a serum immunoelectrophoresis. ${ }^{2}$

\section{Elevated or high-normal parathyroid hormone level}

The parathyroid hormone level is elevated or inappropriately normal (mid-to-high normal range) in patients with primary hyperparathyroidism and accompanying hypercalcemia. In primary hyperparathyroidism, serum phosphate levels are often low because of the phosphaturic effects of parathyroid hormone. Serum phosphate levels may also be low in the presence of a malignant growth that secretes ectopic parathyroid hormone or parathyroid-related peptide. A normal concentration of serum phosphate does not exclude primary hyperparathyroidism or a malignant growth.

Familial hypocalciuric hypercalcemia can also mimic primary hyperparathyroidism and result in elevated levels of both serum calcium and parathyroid hormone or a high-normal nonsuppressed level of parathyroid hormone in the presence of hypercalcemia. Familiar hypocalciuric hypercalcemia is an inherited disorder with autosomal dominant transmission in which a heterozygous mutation inactivates the gene encod-

Table 1: Results of laboratory investigations for a 17-year-old man presenting with thirst, lethargy and fatigue over a one-month period

\begin{tabular}{|lc|}
\hline Test (normal values) & Results \\
\hline lonized calcium, mmol/L (1.17-1.33) & $1.45-1.50$ \\
\hline $24 \mathrm{hr}$ urine calcium, mmol/d (1.2-6.2) & 4.5 \\
\hline $24 \mathrm{hr}$ urine creatinine, mmol/d (5.0-16.0) & 13.0 \\
\hline Serum creatinine, $\mu \mathrm{mol} / \mathrm{L}(45-97)$ & 66 \\
\hline $\begin{array}{l}\text { 25-hydroxyvitamin } \mathrm{D}(<25 \mathrm{nmol} / \mathrm{L}, \text { deficient; }<75 \\
\text { nmol/L, insufficient; }>250 \mathrm{nmol} / \mathrm{L}, \text { toxic) }\end{array}$ & 40 \\
\hline Parathyroid hormone, pmol/L (1.3-7.6) & $5.5-10.3$ \\
\hline Serum phosphate, mmol/L (0.90-1.45) & 1.21 \\
\hline Calcium-to-creatinine clearance ratio & $0.008-0.009$ \\
\hline
\end{tabular}

ing the calcium-sensing receptor. The homozygous form is extremely rare and results in severe hypercalcemia at birth (neonatal severe hyperparathyroidism). Familial hypocalciuric hypercalcemia is associated with an increased set point for serum calcium, which requires higher levels of calcium to suppress the release of parathyroid hormone. Because the mutation also affects the kidneys, renal reabsorption of calcium is enhanced and leads to relative hypocalciuria.

The clearance ratio of calcium to creatinine can help identify the presence of familial hypocalciuric hypercalcemia and exclude primary hyperparathyroidism. About $80 \%$ of patients with familial hypocalciuric hypercalcemia have a clearance ratio of calcium to creatinine of less than 0.01 . The remainder have a clearance ratio of greater than 0.01 , and these values overlap those seen in sporadic primary hyperparathyroidism. ${ }^{5}$

A low clearance ratio can also be seen in patients with vitamin D insufficiency, a very low intake of calcium or chronic kidney disease; these conditions should be excluded before confirming familial hypocalciuric hypercalcemia. Because of the possibility of de novo mutations, an analysis of the gene encoding the calcium-sensing receptor is recommended when the laboratory profile does not exclude familial hypocalciuric hypercalcemia. ${ }^{5}$

Familial hypocalciuric hypercalcemia should be considered in the differential diagnosis of hypercalcemia, particularly in patients younger than 20 years of age and in those with a family history of hypercalcemia. Familial hypocalciuric hypercalcemia is benign and usually presents as an incidental finding in patients who are asymptomatic. Patients with familial hypocalciuric hypercalcemia should not be recommended for parathyroidectomy. ${ }^{5,6}$

Hyperparathyroidism can be mimicked by treatment with certain drugs. Lithium can elevate both parathyroid hormone and serum calcium levels. Elevations in the serum calcium level may also be due to treatment with thiazide diuretics, which increases renal calcium reabsorption. These medications should be stopped if possible, and blood tests should be repeated after three months. ${ }^{5}$

Normocalcemic hyperparathyroidism is diagnosed when an elevated parathyroid hormone level and a consistently normal serum ionized calcium level are seen in the absence of secondary hyperparathyroidism. Causes of secondary hyperparathyroidism may include vitamin D insufficiency, renal impairment or medications. Normocalcemic hyperparathyroidism may be an early form of primary hyperparathyroidism or a state of resistance to parathyroid hormone. The natural history of this condition is not fully understood and requires further study. ${ }^{7}$ 


\section{Suppressed parathyroid hormone levels}

Parathyroid hormone levels are suppressed in individuals with parathyroid-independent hypercalcemia. This includes hypercalcemia secondary to renal failure and to adrenal insufficiency. ${ }^{2}$ Thyrotoxicosis can result in mild hypercalcemia. Myeloma can cause hypercalcemia, as can sarcoidosis and other granulomatous diseases that cause increased production of 1,25-dihydroxyvitamin $\mathrm{D}_{3}$. An increase in alkaline phosphatase in combination with hypercalcemia may be a sign of metastatic skeletal disease or Paget disease of bone and should be evaluated further.

\section{The case revisited}

Our patient had elevated levels of parathyroid hormone and serum calcium (both total and ionized). He was not taking any medications that might have caused hypercalcemia. His calcium to creatinine clearance ratio was less than 0.01 , and thus we considered familial hypocalciuric hypercalcemia as a possible diagnosis. To rule this out, we measured the serum calcium levels in our patient's parents and siblings. The results were normal. An analysis of our patient's DNA was also normal, which ruled out a de novo mutation causing familial hypocalciuric hypercalcemia. The diagnosis was primary hyperparathyroidism.

Box 1: Useful laboratory investigations for suspected hypercalcemia ${ }^{1-3}$

Biochemical analyses of the blood

- Electrolytes

- Calcium (total and ionized)

- Phosphate

- Magnesium

- Liver tests (alanine aminotransferase, aspartate aminotransferase and albumin)

- Alkaline phosphatase

- Renal function (creatinine and blood urea nitrogen)

Hematologic evaluation

- Complete blood count

Other investigations

- Parathyroid hormone

- Thyroid-stimulating hormone

- Thyroxine and triiodothyronine

- Serum immunoelectrophoresis

- 25-hydroxyvitamin D

- Fasting morning cortisol

- Urine concentrations of calcium and creatinine over 24 hours

\section{How is primary hyperparathyroidism managed?}

Primary hyperparathyroidism is usually detected early, before the start of symptoms, with mild hypercalcemia and no history of kidney stones, fragility fractures or pancreatitis. ${ }^{8}$ However, bone density may be low, particularly at the one-third radial site (the anatomic area of the radius that delineates the beginning of the distal third of that bone), which mostly consists of cortical bone. ${ }^{9,10}$ In primary hyperparathyroidism, bone density that has been relatively well maintained has been noted in the lumbar spine, and moderate reductions in bone density have been seen in the femoral neck and in the hips. ${ }^{9}$ The risk of fracture is increased in patients with symptomatic primary hyperparathyroidism; however, in patients for whom the disease is asymptomatic, the risk of fracture is not yet clearly defined.

\section{Genetic testing}

Primary hyperparathyroidism can occur at any age; however, it is more common in patients over the age of 50 years. ${ }^{8}$ Children and young adults in whom primary hyperparathyroidism has been diagnosed should be carefully evaluated to exclude familial syndromes such as multiple endocrine neoplasia types 1 and 2,

\section{Box 2: Differential diagnosis of hypercalcemia based on the patient's level of parathyroid hormone ${ }^{1,3,4}$}

Elevated or inappropriately normal (mid-to-high normal range) levels of parathyroid hormone may indicate the following:

- Primary hyperparathyroidism

- Tertiary hyperparathyroidism

- Familial hypocalciuric hypercalcemia

- Lithium-associated hypercalcemia

- Malignant growth that secretes ectopic parathyroid hormone

Suppressed parathyroid hormone level may indicate the following:

- A malignant growth releasing peptides or cytokines related to parathyroid hormone

- Excessive intake of vitamin D

- Excess production of vitamin D from infectious or noninfectious granulomatous disease

- Vitamin A toxicity

- Hyperthyroidism

- Adrenal insufficiency

- Acute or chronic renal failure

- Loss of mobility

- Use of drugs such as hydrochlorothiazide, omeprazole, theophylline and manganese

- Milk-alkali syndrome 
familial isolated hyperparathyroidism and jaw tumour syndrome. Tests to analyze DNA for genetic abnormalities that can cause the hereditary forms of primary hyperparathyroidism are now available. The selective use of these tests is recommended in the recent guidelines that address the management of primary hyperparathyroidism. ${ }^{5,11,12}$

\section{Treatment options}

Guidelines suggest that surgery be considered for all patients with symptomatic primary hyperparathyroidism. ${ }^{12}$ This includes those patients with substantially elevated calcium levels, as well as those with signs or symptoms of kidney stones or bone disease (e.g., fracture, osteoporosis).

In asymptomatic primary hyperparathyroidism, parathyroidectomy is appropriate when there are no contraindications to surgery. Parathyroidectomy causes the biochemical profile to return to normal and has been associated with improvements in bone density and fracture-free survival, as well as reduction in the risk of fracture. ${ }^{12}$

When surgery is not done, the clinical course of patients with mild asymptomatic primary hyperparathyroidism seems relatively stable. Serum calcium and parathyroid hormone levels and bone density may remain stable for up to 10 years, ${ }^{12}$ at which time bone density may decrease. The disease may progress more quickly in younger patients (age $<50$ years) who are also at an increased risk of complications. ${ }^{12}$

In some patients with mild asymptomatic primary hyperparathyroidism, the disease can be safely managed without parathyroidectomy. ${ }^{12,13}$ However, patients with impaired renal function and glomerular filtration rates below $60 \mathrm{~mL} / \mathrm{min}$ should have surgery, as should patients younger than 50 years of age, as recommended by guidelines. ${ }^{5,12}$ Patients in whom osteoporosis has been diagnosed using densitometric criteria or because of a fragility fracture should also be advised to have a parathyroidectomy owing to their increased risk of fracture. ${ }^{12}$

\section{Imaging}

Imaging before surgery can help guide the surgical approach. ${ }^{8}$ For example, sestamibi imaging with technetium $99 \mathrm{~m}$ detects mitochondrial uptake of the radionuclide tracer in areas of hyperfunctioning tissue. The injected tracer is initially concentrated in normal thyroid and abnormal parathyroid tissues. The concentration in normal thyroid tissue decreases rapidly, leaving behind foci of relatively enhanced uptake of the tracer in abnormal thyroid and parathyroid tissues. ${ }^{14}$ Sestamibi imaging can, however, miss small adenomas and hyperplasia. A lack of retention of the tracer does not exclude the diagnosis of primary hyperparathyroidism. ${ }^{12} \mathrm{Com}$ puted tomography scans and ultrasonography of the neck can also be helpful tools. A diagnosis of hyperparathyroidism should not be based on imaging alone. Rather, imaging can be done to help guide surgery. ${ }^{12}$

\section{Parathyroidectomy}

New surgical approaches include minimally invasive parathyroidectomy, which can decrease the length of time the surgery takes so that it can be done on an outpatient basis. Completing an intraoperative parathyroid hormone assay after removing the adenoma will confirm that the abnormal parathyroid tissue has been adequately resected and that full exploration of the neck will not be necessary. ${ }^{15}$ The minimally invasive approach is as effective as total open parathyroidectomy. ${ }^{15}$ This procedure should be done by a surgeon who is expert in this complicated technique.

\section{Nonsurgical options for treatment}

Correcting any vitamin D insufficiency and carefully monitoring serum calcium levels is recommended for all patients. ${ }^{12,16}$ Vitamin D insufficiency is often seen with primary hyperparathyroidism and could be a sign of the increased catabolism of 25-hydroxyvitamin $\mathrm{D}$ in the presence of elevated levels of parathyroid hormone. A concomitant vitamin D insufficiency has been associated with higher levels of parathyroid hormone, increased weight of parathyroid adenomas and lower cortical bone densities. ${ }^{5}$ Recently, Grey and colleagues gave 21 women with primary hyperparathyroidism and coexisting vitamin $\mathrm{D}$ insufficiency supplements containing 50000 IU of cholecalciferol (vitamin $\mathrm{D}_{3}$ ) on a weekly basis for 4 weeks, followed by monthly administration for 11 months. Levels of parathyroid hormone decreased by $26 \%$ and serum calcium levels remained stable and did not rise, even with supplementation of cholecalciferol. ${ }^{17}$

Other treatment options include bisphosphonates, hormone replacement therapy, raloxifene and cinacalcet. ${ }^{16}$ These agents have been evaluated in short-term trials. Bisphosphonates and hormone replacement therapy are both effective in improving bone density, but neither lowers serum calcium or parathyroid hormone levels. ${ }^{16,18}$ Data on the efficacy of raloxifene treatment are limited ${ }^{16}$ Cinacalcet, a calcimimetic, increases the sensitivity of the calcium-sensing receptor to calcium and lowers the level of parathyroid hormone; in effect, this treatment is a "chemical parathyroidectomy" and is able to effectively lower the levels of serum calcium and parathyroid hormone in patients with primary hyper- 
parathyroidism. ${ }^{16}$ Improvements in bone density have not yet been seen with cinacalcet, and biomarkers of bone turnover may increase. ${ }^{16}$

There are currently no data available on the risk of fracture with any of these nonsurgical options. These options may be considered for individual patients by targeting skeletal protection with antiresorptive therapy and using cinacalcet to lower the serum calcium level.

\section{Conclusion}

Hypercalcemia should be evaluated carefully, particularly in younger patients in whom hereditary disorders are more common. The condition may be a component of multiple endocrine abnormalities. Primary hyperparathyroidism is a common cause of hypercalcemia, particularly in patients who present in the ambulatory setting. With widespread testing for calcium levels, primary hyperparathyroidism is now being identified during asymptomatic stages. Advances in surgical and medical options for therapy can reduce the risk of fracture and other complications in these patients.

\section{References}

1. Jacobs TP, Bilezikian JP. Rare causes of hypercalcemia. J Clin Endocrinol Metab 2005;90:6316-22.

2. Kronenberg HM, Shlomo M, Polonsky KS, et al. Williams textbook of endocrinology. 11th ed. Philadelphia (PA): Elsevier; 2008.

3. Shour R, Khan A. Approach to the patient with hypercalcemia: diagnostic principles. Diagnosis 2009;26:55-9.

4. Khan A, Bilezikian J. Primary hyperparathyroidism: pathophysiology and impact on the bone. CMAJ 2000;163:184-7.

5. Eastell R, Arnold A, Brandi ML, et al. Diagnosis of asymptomatic primary hyperparathyroidism: proceedings of the third international workshop. J Clin Endocrinol Metab 2009;94:340-50.

6. Fuleihan GE. Familial benign hypocalciuric hypercalcemia. $J$ Bone Miner Res 2002;17:N51-N56.

7. Silverberg SJ, Lewiecki EM, Mosekilde L, et al. Presentation of asymptomatic primary hyperparathyroidism: Proceedings of the Third International Workshop. J Clin Endocrinol Metab 2009; 94:351-65.

8. Asymptomatic primary hyperparathyroidism: standards and guidelines for diagnosis and management in Canada: Consensus
Development Task Force on Diagnosis and Management of Asymptomatic Primary Hyperparathyroidism position paper. Endocr Pract 2003;9:400-5.

9. Silverberg SJ, Shane E, Jacobs TP, et al. A 10-year prospective study of primary hyperparathyroidism with or without parathyroid surgery. N Engl J Med 1999;341:1249-55.

10. Performing and analyzing the forearm scan. In: $Q D R$ series user's guide. Bedford (MA): Hologic; 2005. p. 10-17.

11. Brandi ML, Gagel RF, Angeli A, et al. Guidelines for diagnosis and therapy of MEN type 1 and type 2. J Clin Endocrinol Metab 2001;86:5658-71.

12. Bilezikian JP, Khan AA, Potts JT Jr. Third International Workshop on the Management of Asymptomatic Primary Hyperthyroidism: guidelines for the management of asymptomatic primary hyperparathyroidism: summary statement from the Third International Workshop. Clin Endocrinol Metab 2009;94:335-9.

13. Khan AA, Bilezikian JP, Potts JT Jr.; guest editors for the Third International Workshop on Asymptomatic Primary Hyperparathyroidism. The diagnosis and management of asymptomatic primary hyperparathyroidism revisited. J Clin Endocrinol Metab 2009;94:333-4.

14. Gotway MB, Leung JWT, Gooding GA, et al. Hyperfunctioning parathyroid tissue: spectrum of appearances on noninvasive imaging. AJR Am J Roentgenol 2002;179:495-502.

15. Udelsman R, Pasieka JL, Sturgeon C, et al. Surgery for asymptomatic primary hyperparathyroidism: proceedings of the third international workshop. J Clin Endocrinol Metab 2009;94:366-72.

16. Khan A, Grey A, Shoback D. Medical management of asymptomatic primary hyperparathyroidism: proceedings of the third international workshop. Clin Endocrinol Metab 2009;94:373-81.

17. Grey A, Lucas J, Horne A, et al. Vitamin D repletion in patients with primary hyperparathyroidism and coexistent vitamin D insufficiency. J Clin Endocrinol Metab 2005;90:2122-6.

18. Khan AA, Bilezikian JP, Kung AW, et al. Alendronate in primary hyperparathyroidism: a double blind, randomized, placebo controlled trial. J Clin Endocrinol Metab 2004;89:3319-25.

Affiliations: From the Department of Medicine, McMaster University, Hamilton, Ont.

Contributors: Both of the authors shared responsibility for the conception, design and drafting of this article, revised it critcally for important intellectual content and approved the final version submitted for publication.

Primer articles are brief narrative reviews on interesting, important and timely clinical topics. The articles emphasize clinical application and are based on the best evidence available. Author instructions are available at cmaj.ca. Submit Primers (maximum 1500 words and up to 10 references) to http://mc.manuscriptcentral.com/cmaj or emaildiane.kelsall@cmaj.ca to discuss ideas. 